\title{
Association Between Tic Aggravation and Methylphenidate in Youth With Attention-Deficit/Hyperactivity Disorder
}

\author{
Jung Yoon Cha, Yoo-Sook Joung ${ }^{\bowtie}$, Soohwan Oh, Byung Wook Kim, In Mok Song, and Bo Mi Ahn \\ Department of Psychiatry, Samsung Medical Center, Sungkyunkwan University School of Medicine, Seoul, Republic of Korea
}

\begin{abstract}
Objective This study aimed to determine the tic aggravation event rate and cumulative incidence rate in the use of methylphenidate $(\mathrm{MPH})$ treatment in attention-deficit/hyperactivity disorder (ADHD) and the factors that influence tic aggravation.

Methods We conducted a retrospective chart review of children and adolescents aged between 6 to 15 years, who were diagnosed with ADHD from January 2017 to December 2019. A total of 121 subjects were included. The MPH dosage, psychiatric family history, comorbidity and past history of tics were assessed through chart review and the psychological examinations data were included. Collected data were analyzed using Cox regression and Kaplan-Meier survival analysis.

Results Tic aggravation event rates without a past history of tics were $2.9 \%$ with MPH treatment in ADHD. Past history of tics, total $\mathrm{MPH}$ dosage and age were the factors associated with tic aggravation ([HR 21.46, $\mathrm{p}<0.001]$, [HR 0.94, $\mathrm{p}=0.023]$, [HR $0.79, \mathrm{p}=0.021]$ for each). Cumulative incidence of tic aggravation was different between groups with or without past tic history. When treated with MPH, all tic aggravation appeared within approximately eight months but for subjects with a past history of tic, aggravation showed within approximately six months $(\mathrm{p}<0.001)$.

Conclusion Tic aggravation event rate was significantly low especially in the group without a past history of tics with the use of MPH in ADHD. However, a thorough assessment of past history of tics, and close monitoring during the first six-eight months of treatment with MPH is needed to avert a potential worsening of tics.

Psychiatry Investig 2021;18(9):818-824
\end{abstract}

Keywords Attention-deficit/hyperactivity disorder; Tic; Methylphenidate; Cumulative incidence.

\section{INTRODUCTION}

Attention-deficit/hyperactivity disorder (ADHD) is one of the most common children and adolescents psychiatric disorders, with an estimated prevalence of $2 \%-9.5 \%{ }^{1-3}$ Approximately $20 \%$ of children with ADHD develop a chronic tic disorder. ${ }^{4}$ Children with ADHD and comorbid tics have a lower psychosocial function, academic performance, peer relationships, and executive functioning than those with ADHD or tics alone. ${ }^{5,6}$ Also, the risk of aggressive and delinquent behavior and conduct difficulties in tics are largely posed by the presence of ADHD. ${ }^{7}$ Due to the impact of ADHD on tic disorder, ADHD symptoms treatment is prioritized over the medical treatment of tics. ${ }^{8}$

\footnotetext{
Received: February 5, 2021 Revised: May 4, 2021

Accepted: July 13, 2021

$\triangle$ Correspondence: Yoo-Sook Joung, MD, PhD

Department of Psychiatry, Samsung Medical Center, Sungkyunkwan University School of Medicine, 81 Irwon-ro, Gangnam-gu, Seoul 06351, Republic of Korea Tel: +82-2-3410-3589, Fax: +82-2-3410-0050, E-mail: yschoung@skku.edu

(a) This is an Open Access article distributed under the terms of the Creative Commons Attribution Non-Commercial License (https://creativecommons.org/licenses/bync/4.0) which permits unrestricted non-commercial use, distribution, and reproduction in any medium, provided the original work is properly cited.
}

In ADHD, psychostimulants such as methylphenidate (MPH) are commonly used, and recommended as the firstline pharmacologic treatment for ADHD. ${ }^{9}$ However, the possibility of worsening tics with the use of stimulant medications has restricted the use of MPH in children with ADHD and comorbid tics. ${ }^{10}$ On contrary, multiple randomized controlled trials have demonstrated that MPH did not worsen tics in the majority of children and was, in fact, beneficial in treating ADHD symptoms in children with ADHD and comorbid tics. ${ }^{8}$ Also, MPH demonstrated efficacy in improving ADHD symptoms in children with comorbid tics and no evidence was found that MPH worsened tic severity in the short term. ${ }^{11}$ Despite the new evidence that this relationship was temporal and not causal, pharmacologic treatment of children with ADHD comorbid with tics is still a controversial topic and a challenge for the clinicians. ${ }^{8}$

Usually ADHD symptoms appears about 2 to 3 years before the tics when ADHD and tics co-occur in an individual. ${ }^{12}$ Therefore, it is difficult to determine the contribution of stimulants medication on tics development; whether the tics naturally developed regardless of stimulant use or the tics were 
the side effect of stimulants. A cardinal feature of tics waxing and waning in severity, would also make it difficult to determine whether tics developed were a side effect of stimulants medication. ${ }^{13}$

The objective of this study was to determine the probability of tic aggravation with the use of MPH, and the factors that were associated with tic aggravation when using MPH. In addition, we aimed to determine the cumulative incidence of tic aggravation when using MPH in ADHD, and give suggestions of important close monitoring periods during which tic aggravation worsens.

\section{METHODS}

\section{Subjects and study design}

Our subjects were Koreans, aged between 6 to 15 years, who visited the Department of Psychiatry at Samsung Medical Center, located in Seoul, from January 1, 2017 to December 31, 2019 for the first time. Each subject met Diagnostic and Statistical Manual of Mental Disorders, Fifth Edition (DSM-5) criteria for ADHD.

The exclusion criteria was: 1) diagnosed with tic disorder before first visit, 2) IQ score lower than 50 according to the KWISC-IV, 3) have comorbid autism spectrum disorder (ASD) or organic brain disease, 4) follow-up period shorter than 6 months, and 5) not treated with MPH during the follow-up period. As tic aggravation had hyperkinetic movements which can be commonly observed in children with ASD, ${ }^{14}$ the symptom similarity and linkage between complex tics and the repetitive and restrictive behavior that are of diagnostic significance for ASD could make it difficult to clarify the main outcome of tic aggravation. Therefore, we excluded ASD from study population. As our clinical center is university general hospital in Korea, many ADHD patients first visit our center just for second clinician opinion or psychological inspection not for pharmacological treatment. Also, if patients needed to have treatment around their hometown, they were transferred to short distance hospital. Therefore, most of new patients diagnosed with ADHD had only once or twice visit and those subjects were not adequate for this study.

The study was based on retrospective chart review and received ethical approval from the Samsung Medical Center Institutional Review Board (IRB No. SMC 2020-09-197-001).

\section{Measurement and assessment}

Psychiatric diagnosis was described on electronic medical records by certified child and adolescent psychiatrists. To figure out the factors that were associated with tic aggravation when using MPH, we compared the characteristics of the tic aggravation group and the non-tic aggravation group. We evaluated the usage of MPH and the subjects treated with both MPH and atomoxetine (ATM) simultaneously were excluded for a clear evaluation of medication effect. If subjects had changed medication, MPH to ATM or ATM to MPH during the follow-up period, cross-over data were included only if any such treatment had been discontinued for at least 2 weeks before changing. The maximal dosage of MPH (mg) and maximal dosage per body weight $(\mathrm{mg} / \mathrm{kg})$ were evaluated. Psychiatric family history (parents, siblings, and grandparents) and comorbidity were assessed by certified psychiatrists based on a chart review that met DSM- 5 criteria. The subjects that had shown tic symptoms without a diagnosis of tic disorder before the first visit to our center or just showed up tic symptoms on their first visit were sorted as a history of tics positive group. Subjects with no history of tic symptoms ever were sorted as history of tics negative group. Psychological examinations including the Korean version of Wechsler Intelligence Scale for Children 4th edition(K-WISC-IV) and the Korean version of the ADHD Rating Scale (K-ARS) ${ }^{15}$ were conducted by certified psychologists and psychiatrists. The Full-Scale Intelligence Quotient (FSIQ) was collected using K-WISC-IV. Although intellectual disability is not defined solely by low FSIQ score in IQ test according to DSM-5, for the clarifying data, we classified FSIQ below 70 as a mild intellectual disability and under 50 as a moderate intellectual disability according to DSM-IVTR. Most previous treatment trials for ADHD have excluded children with intellectual disability, partly because children with intellectual disability often have coexisting medical problems and are also less likely to self-report adverse events. ${ }^{16}$ Furthermore, clinical response was more heterogeneous in children with severe mental retardation than in children with IQ above 50 , and children with mental retardation and ADHD may be more prone to adverse effects than typically developing children with ADHD. ${ }^{17}$ However, moderate intellectual disability is known as having organic etiologies, while, mild intellectual disability could be etiologically idiopathic. ${ }^{18,19}$ Therefore, we tried to minimize heterogeneity of results and minimize brain organic vulnerability by excluding subjects with moderate intellectual disability. To figure out the difference between group without moderate intellectual disability and group without mild and moderate intellectual disability, we conducted subgroup analysis between two groups. We grouped K-ARS score into three categories; total score, inattention score, and hyperactivity/impulsivity score. Combination drugs such as antidepressants, atypical antipsychotics and mood stabilizer were evaluated, and especially atypical antipsychotics were evaluated by drug component such as aripiprazole, quetiapine and risperidone.

Tic aggravation was defined as new onset of tic symptoms or worsening of existing tics. The status of tic aggravation was 
evaluated using chart records such as a description of tic aggravation, discontinuation, or change of the medication due to tic aggravation. Tic aggravation was assessed during the follow-up period and the date of tic aggravation was noted.

\section{Data analysis}

Fisher's exact test or Wilcoxon rank-sum test were performed for comparisons between the tic aggravation group and nontic aggravation group with respect to age, gender, MPH dosage, psychiatric family history, comorbidity, past history of tics, FSIQ and K-ARS score. Cox proportional hazard analysis was performed to assess the associations between tic aggravation and other factors; results were estimated as hazard ratios (HRs) with 95\% confidence intervals (CIs). Multivariable Cox regression was performed for models adjusted for age, MPH total dosage and past history of tics. Because there were multiple events due to treatment change for some subjects, we used a Cox proportional hazard regression model with clusters. A Kaplan-Meier survival analysis was conducted to compare survival rates between groups with and without past history of tics. The Statistical Package for the Social Sciences (SPSS) version 25.0 (IBM Corp., Armonk, NY, USA) was used for these statistical analyses. The p-value $<0.05$ was considered significant.

\section{RESULTS}

A total of 578 subjects who had visited our center and diagnosed with ADHD were included in the present study. Among them, 15 had already been diagnosed with tic disorder before the first visit, one had their IQ below 50, 5 had comorbidity of ASD and two had organic brain disease such as brain tumor and receiving chemotherapy, 372 had a short follow-up period (less than 6 months) and 66 had not received MPH during follow-up period; therefore they were excluded. The reasons why several subjects were not treated with MPH were underweight, mild symptoms, and their caregivers refused to give the medication. Excluding combination therapy data of MPH and ATM while including cross-over medication data, a total of 121 eligible data (mean age: $9.57 \pm 2.9$ years) were sorted.

The overall tic aggravation event rates when using MPH were $8.3 \%$. We evaluated and compared the baseline characteristics of tic aggravation group and non-tic aggravation group. The mean age of tic aggravation group was 7.90 years $( \pm 1.37)$ and that of non-tic aggravation group was 9.73 years $( \pm 2.97)$. All subjects who showed tic aggravation were male. Total maximal dosage of MPH was $25.50 \mathrm{mg}( \pm 11.65)$ in tic aggravation group and $36.86 \mathrm{mg}( \pm 15.53)$ in non-tic aggravation group, which showed difference between groups ( $\mathrm{p}=0.029$ ). Concerning psychiatric family history, in tic aggravation group, one subject had a family history (father with a tic disorder) and in non-tic aggravation group, 17 subjects had psychiatric family histories (Supplementary Material [A] in the onlineonly Data Supplement). There was no significant difference between the two groups' overall psychiatric family history $(\mathrm{p}=1.000)$ and tic disorder family history $(\mathrm{p}=0.083)$. In the tic aggravation group, 6 subjects had comorbidities and in the non-tic aggravation group, 40 subjects had comorbidities (Supplementary Material [B] in the online-only Data Supplement); there was no significant difference between the two groups ( $\mathrm{p}=0.177$ ). There was a significant difference between the event rate of tic aggravation in the group with a past history of tics and the group without a past history of tics ( $\mathrm{p}<$ $0.001) ; 7$ out of 19 (36.8\%) who had a past history of tics showed tic aggravation, and 3 out of 102 (2.9\%) who did not have past history of tics showed tic aggravation. The mean FSIQ of tic aggravation group was $90.10( \pm 16.79)$ and non-tic aggravation group was $87.05( \pm 19.43)$ and there was no significant difference between two groups. To investigate the influence of intellectual disability degree on tic aggravation, we compared groups including mild intellectual disability (FSIQ $\geq 50$ ) and groups without mild intellectual disabililty (FSIQ $\geq 70$ ), and there was no significant difference between groups in subgroup analysis. The total K-ARS score was higher in tic aggravation group though statistically not significant. Several subjects were treated with combination therapy considering their comorbidities. The combination drugs were antidepressants ( $n=13)$, atypical antipsychotics $(n=24)$, mood stabilizer $(\mathrm{n}=5)$ and some of them were used in complex. In our study, there were subjects who had been treated with aripiprazole $(\mathrm{n}=21)$, quetiapine $(\mathrm{n}=5)$, risperidone $(\mathrm{n}=3)$. The atypical antipsychotics such as aripiprazole, risperidone and quetiapine could be used to manage tic disorder, ${ }^{20}$ however in this study, there was no significant difference between the event rate of tic aggravation in the group with previous antipsychotics use and the group without previous antipsychotics use $(\mathrm{p}=0.670)$. Subjects were already using antipsychotics due to other comorbidities such as oppositional defiant disorder, disruptive mood dysregulation disorder and bipolar disorder. It was difficult to admit that atypical antipsychotics definitely influenced on tic aggravation in our study, hence we excluded further data analysis in our study (Table 1).

The association between tic aggravation and multiple factors when using MPH was demonstrated by Cox proportional hazard analysis (Table 2). In univariate analysis, a past history of tics showed a significantly increased HR (HR 16.03; 95\% CI, 4.20-61.20; $\mathrm{p}<0.001$ ). The age (HR 0.78; 95\% CI, 0.66$0.92 ; \mathrm{p}=0.004)$ and $\mathrm{MPH}$ total dosage (HR $0.94 ; 95 \% \mathrm{CI}, 0.90^{-}$ $0.99 ; \mathrm{p}=0.014)$ also showed significant difference in tic aggravation. After adjusting for age and MPH total dosage, the past 
JY Cha et al.

Table 1. Baseline characteristics of subjects treated with $\mathrm{MPH}(\mathrm{N}=121)$

\begin{tabular}{|c|c|c|c|}
\hline Variables & $\begin{array}{c}\text { Tic aggravation }(-) \\
(\mathrm{N}=111)\end{array}$ & $\begin{array}{l}\text { Tic aggravation }(+) \\
\quad(\mathrm{N}=10)\end{array}$ & $\mathrm{p}$-value \\
\hline Age of years & $9.73(2.97)$ & $7.90(1.37)$ & $0.098^{*}$ \\
\hline Gender & & & $0.207^{\dagger}$ \\
\hline Male & $90(81.1)$ & $10(100.0)$ & \\
\hline Female & $21(18.9)$ & $0(0.0)$ & \\
\hline \multicolumn{4}{|l|}{ Methylphenidate dosage } \\
\hline Total dosage mg & $36.86(15.53)$ & $25.50(11.65)$ & $0.029 *$ \\
\hline $\mathrm{mg} / \mathrm{kg}$ & $0.87(0.33)$ & $0.72(0.31)$ & $0.258^{*}$ \\
\hline Psychiatric family history & & & $1.000^{\dagger}$ \\
\hline None & $94(84.7)$ & $9(90.0)$ & \\
\hline 1 or more & $17(15.3)$ & $1(10.0)$ & \\
\hline Comorbidity & & & $0.177^{\dagger}$ \\
\hline None & $71(64.0)$ & $4(40.0)$ & \\
\hline 1 or more & $40(36.0)$ & $6(60.0)$ & \\
\hline Past history of tics & & & $<0.001^{\dagger}$ \\
\hline No & $99(89.2)$ & $3(30.0)$ & \\
\hline Yes & $12(10.8)$ & $7(70.0)$ & \\
\hline FSIQ & $87.05(19.43)$ & $90.10(16.79)$ & $0.546^{*}$ \\
\hline \multicolumn{4}{|l|}{ K-ARS } \\
\hline Total score & $22.49(12.08)$ & $22.80(13.27)$ & $0.996^{*}$ \\
\hline Inattention & $14.10(5.84)$ & $12.33(5.43)$ & $0.260^{*}$ \\
\hline Hyperactivity/impulsivity & $9.90(6.05)$ & $13.00(6.22)$ & $0.122^{*}$ \\
\hline Previous antipsychotics use & & & $0.670^{\dagger}$ \\
\hline No & $93(83.8)$ & $8^{\ddagger}(80)$ & \\
\hline Yes & $18(16.2)$ & $2(20)$ & \\
\hline
\end{tabular}

Data are presented as mean (SD) or N (\%). *median (IQR), Wilcoxon rank sum test; ${ }^{\dagger}$ Fisher’s exact test; $¥ 4$ subjects had used antipsychotics after tic aggravation. MPH, Methylphenidate; SD, standard deviation; N, number; FSIQ, Full Scale Intelligence Quotient; IQR, interquartile range; K-ARS, Korean version of the attention-deficit/hyperactivity disorder

history of tics showed increased HR (HR 21.46; 95\% CI, 5.0291.85; $\mathrm{p}<0.001)$. The age showed similar HR after adjusting for MPH total dosage and past history of tic (HR 0.79; 95\% CI, 0.64-0.97; $\mathrm{p}=0.021$ ), and likewise, MPH total dosage showed similar HR after adjusting for age and past history of tics (HR 0.94; 95\% CI, 0.89-0.99; $\mathrm{p}=0.023$ ) also which still demonstrated significant difference (Table 3). Additionally, in the tic aggravation group, there was $70 \%$ of tic resolution and $30 \%$ of tic persistence after MPH discontinuation.

Through Kaplan-Meier plot, we evaluated the difference in the onset of tic aggravation events between groups with and without past history of tics. On Kaplan-Meier survival curves, the cumulative incidence of tic aggravation of past history of tics positive group was significantly higher than past history of tics negative group $(p<0.001)$. When first treated with MPH, all the tic aggravation events appeared within 8.33 months and the subjects with past history of tics showed tic aggrava- tion within 6.16 months (Figure 1 and Supplementary Figure 1 in the online-only Data Supplement). In other words, regardless of a past history of tics, all the tic aggravation events appeared within approximately 8 months, highlighting the need to look out for worsening of tics relatively early in the MPH treatment. Especially, the subjects with a past history of tics showed tic aggravation within about 6 months, emphasizing the need to look out for worsening of tics more intensely.

\section{DISCUSSION}

The treatment for ADHD must be based on a comprehensive diagnostic evaluation and should consider the age, severity of symptoms, duration of illness and a balance between the positive effects and adverse effects. Therefore, before initiating pharmacological treatment, drug efficacy and adverse events should be evaluated and detailed psychiatric history 
Table 2. Association between tic aggravation and multiple factors using methylphenidate analyzed by Cox proportional hazard analysis

\begin{tabular}{lcc}
\hline & $\begin{array}{c}\text { Hazard ratio } \\
(95 \% \mathrm{CI})^{*}\end{array}$ & p-value \\
\hline Age & $0.78(0.66-0.92)$ & 0.004 \\
Male $^{\dagger}$ & $>999.999$ & $<0.001$ \\
Methylphenidate dosage & & \\
$\quad$ Total dosage & $0.94(0.90-0.99)$ & 0.014 \\
$\quad$ mg/kg & $0.22(0.03-1.74)$ & 0.152 \\
Psychiatric family history & $0.64(0.08-4.89)$ & 0.667 \\
Comorbidity & $2.63(0.75-9.20)$ & 0.131 \\
Past history of tics & $16.03(4.20-61.20)$ & $<0.001$ \\
FSIQ & $1.01(0.98-1.04)$ & 0.444 \\
K-ARS & & \\
$\quad$ Total score & $1.00(0.95-1.06)$ & 0.968 \\
Inattention & $0.95(0.86-1.05)$ & 0.316 \\
Hyperactivity/impulsivity & $1.07(0.98-1.17)$ & 0.114 \\
\hline
\end{tabular}

*hazard ratio and $95 \% \mathrm{CI}$ estimated by Cox proportional risk mod$\mathrm{el} ;{ }^{\dagger}$ compared with female sex. CI, confidence interval

Table 3. Association between tic aggravation and adjusted factors with using methylphenidate by multivariable Cox analysis

\begin{tabular}{lcc}
\hline & $\begin{array}{c}\text { Hazard ratio } \\
(95 \% \mathrm{CI})^{*}\end{array}$ & p-value \\
\hline Age & $0.79(0.64-0.97)$ & 0.021 \\
Methylphenidate total dosage & $0.94(0.89-0.99)$ & 0.023 \\
Past history of tics & $21.46(5.02-91.85)$ & $<0.001$ \\
\hline
\end{tabular}

*hazard ratio and $95 \%$ CI estimated by Cox proportional risk model. CI, confidence interval

from caregivers and families should be taken.

In the present study, approximately $8.3 \%$ of subjects treated with MPH showed tic aggravation, and $70 \%$ of them had a past history of tics. There was a significant difference in the event rate of tic aggravation between ADHD patients with a past history of tics and those without; the event rate of tic aggravation among subjects diagnosed with $\mathrm{ADHD}$, with no a past history of tics and treated with MPH was $2.9 \%$ while ADHD patients with a past history of tics and treated with MPH showed a significantly higher event rate of $36.8 \%$.

In this study, tic aggravation group was comprised of male gender only. As ADHD and tic disorder were more commonly diagnosed in males, ${ }^{21-25}$ our study also showed higher proportion of subjects with male gender. In tic aggravation group, subjects were younger than in non-tic aggravation group though statistically not significant. The result showed that the younger the subjects, the higher the tic aggravation rate (HR 0.79). Because the parents who brought their children to our hospital were aware of the disease and highly motivated to be in-

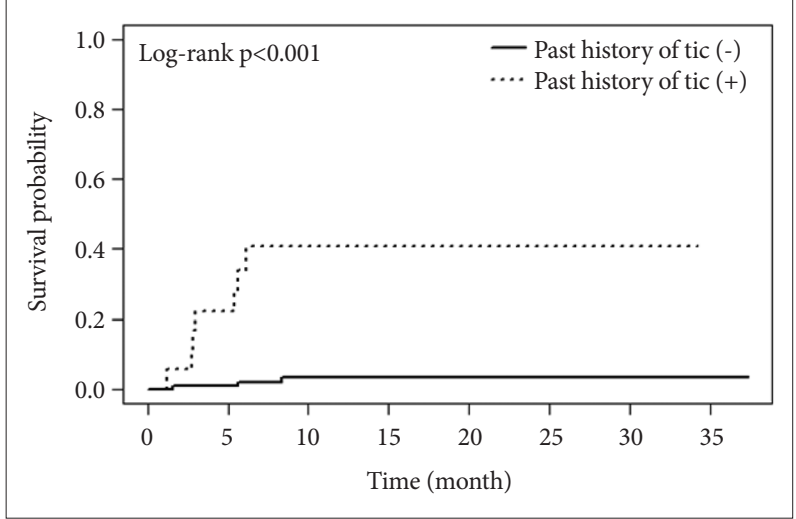

Figure 1. Kaplan-Meier survival curve to compare tic aggravation event rate between groups with and without past history of tics.

volved in the management of their children's symptoms, they might have higher chances of detecting children's tic symptoms in early age. Additionally, stimulants have differing effects on the neuronal substrate at different ages and the 'neuronal imprinting theory' suggests a differential effect of $\mathrm{MPH}$ exposure, dependent on sensitive periods in brain development, in that treatment in younger childhood can have different effects on the brain and its function. ${ }^{26}$ Therefore, there might be influence in tic aggravation according to age. In a previous study, there was no difference in tic severity with different MPH dosages, ${ }^{27}$ however, this study showed that as MPH total dosage increases, event rate of tic aggravation reduces. Because tic worsened earlier during follow-up periods, clinicians were hesitant to use high doses of MPH and instead discontinued or switch to a different medication. That may be a possible reason that MPH total dosage and tic aggravation showed an inverse relationship.

There was no significant difference in tic aggravation event rates in overall psychiatric family history analysis. Most of the psychiatric family histories included in this study were depressive disorder and ADHD. As genetic loading plays a leading role in the tics etiology ${ }^{28}$ we assessed if tic family history had any association with tic aggravation. There was no significant difference between groups with a tic family history and those without, however, accurate evaluation would have been limited by the small sample size. In the present study, comorbidity showed no significant influence on tic aggravation. Similar to other studies, the most common comorbidities in our study were anxiety disorder, oppositional defiant disorder and depressive disorder. ${ }^{29,30}$ Though not statistically significant, FSIQ was higher in tic aggravation group than in the non-tic aggravation group.

The past history of tics showed significant impact on tic aggravation. Even when assuming the lowest risk of tic aggravation at the lower bound of the $95 \%$ CI, a past history of tics was 5 -fold more likely to increase tic aggravation which was 
fairly powerful. Thus, these results suggest that it is important to assess ADHD patients for a past history of tics. Therefore, thorough assessment of past history of tics is needed and a close monitoring until 6 months in case of a past history of tics is important in ADHD patients treated with MPH. Additionally, because clinician might hesitate to start MPH or use high dosage of MPH to subjects with past history of tics, tic aggravation risk on subjects with past history of tics may be underestimated and further clinical studies are needed for the future.

There were several limitations to this study that might have affected our findings. First, evaluating of the factors influencing tic aggravation was limited by the small sample size; therefore, further studies with larger sample sizes are needed. Second, the selective reporting from parents or caregivers could have led to selective bias and therefore influenced on inaccurate results. To minimize this bias, each subject was interviewed by two doctors and the data was summed up for thorough examination. Third, there was inclusion of MPH and ATM cross-over data. Cross-over data were included only if either of the treatment had to be discontinued for at least 2 weeks before changing to a different one, this period would be sufficient wash out time to eliminate any effects of the previous medication. Fourth, the heterogeneity of the data used to assess tic aggravation was a potential limitation. There was no standardized data scale to evaluate tic aggravation, therefore, assessment relied on different reports; some relied on parents or caregivers whereas others relied on direct subjects observation. In addition, tics have been known to worsening during periods of stress, excitement and fatigue, ${ }^{31}$ it was difficult to demonstrate whether tic aggravation was caused by MPH or waxing and waning of tic themselves. Fifth, we overlooked drug interactions that could result from combined therapy, especially atypical antipsychotics. There was no significant difference in tic aggravation regarding previous antipsychotics use $7.9 \%$ of subjects without previous antipsychotics use showed tic aggravation and $10 \%$ of subjects with previous antipsychotics use showed tic aggravation. Furthermore, because of the limitation of this retrospective study, future prospective studies should be done to avert the possible influence on antipsychotics to tic symptoms. Sixth, we excluded missing data of all the categories and that could have led to an inflated estimation and selection bias.

In conclusion, based on our findings, tic aggravation event rate was quite low in the use of MPH in ADHD children and adolescents, especially when they did not have a past history of tics. However, a thorough assessment of past history of tics, and close monitoring during the first six-eight months of treatment with MPH is needed to avert potential worsening of tics. The strength of this study is that it demonstrates the factors as- sociated with tic aggravation during ADHD treatment with $\mathrm{MPH}$ and further recommends cautious observation of periods during which tics worsen by cumulative incidence. Our study did not provide a causal relationship between factors and tic aggravation, and the definite time of close monitoring, therefore, further prospective research and replication study is needed in the future.

\section{Supplementary Materials}

The online-only Data Supplement is available with this article at https://doi.org/10.30773/pi.2021.0047.

\section{Availability of Data and Material}

All data generated or analyzed during the study are included in this published article (and its supplementary information files).

\section{Conflicts of Interest}

The authors have no potential conflicts of interest to disclose.

\section{Author Contributions}

Conceptualization: Yoo-Sook Joung, Jung Yoon Cha. Data curation: Jung Yoon Cha. Formal analysis: Jung Yoon Cha, Soohwan Oh. Investigation: Jung Yoon Cha, Byung Wook Kim. Methodology: Yoo-Sook Joung, Bo Mi Ahn. Project administration: Yoo-Sook Joung. Supervision: Yoo-Sook Joung. Writing_original draft: Jung Yoon Cha, In Mok Song. Writingreview \& editing: Yoo-Sook Joung.

\section{ORCID iDs}

Jung Yoon Cha https://orcid.org/0000-0003-2207-9197

Yoo-Sook Joung https://orcid.org/0000-0002-9225-4643

Soohwan Oh https://orcid.org/0000-0002-7881-2215

Byung Wook Kim https://orcid.org/0000-0001-7094-6123

In Mok Song https://orcid.org/0000-0002-0998-8284

Bo Mi Ahn https://orcid.org/0000-0002-6086-1975

\section{Funding Statement}

None.

\section{REFERENCES}

1. Feldman HM, Reiff MI. Clinical practice. Attention deficit-hyperactivity disorder in children and adolescents. N Engl J Med 2014;370:838846.

2. Felt BT, Biermann B, Christner JG, Kochhar P, Harrison RV. Diagnosis and management of ADHD in children. Am Fam Physician 2014;90: 456-464.

3. Floet AM, Scheiner C, Grossman L. Attention-deficit/hyperactivity disorder. Pediatr Rev 2010;31:56-69.

4. Gabbard GO. Treatments of Psychiatric Disorders (2nd ed). Arlington, VA, US: American Psychiatric Association, 1995, p. lxxxvi, 2472-lxxxvi, 2472.

5. Spencer T, Biederman J, Harding M, O’Donnell D, Wilens T, Faraone S, et al. Disentangling the overlap between Tourette's disorder and ADHD. J Child Psychol Psychiatry 1998;39:1037-1044.

6. Bawden HN, Stokes A, Camfield CS, Camfield PR, Salisbury S. Peer relationship problems in children with Tourette's disorder or diabetes mellitus. J Child Psychol Psychiatry 1998;39:663-668.

7. Cavanna AE, Servo S, Monaco F, Robertson MM. The behavioral spectrum of Gilles de la Tourette syndrome. J Neuropsychiatry Clin Neurosci 2009;21:13-23.

8. Osland ST, Steeves TD, Pringsheim T. Pharmacological treatment for 
attention deficit hyperactivity disorder (ADHD) in children with comorbid tic disorders. Cochrane Database Syst Rev 2018;6:Cd007990.

9. Wolraich M, Brown L, Brown RT, DuPaul G, Earls M, Feldman HM, et al. ADHD: clinical practice guideline for the diagnosis, evaluation, and treatment of attention-deficit/hyperactivity disorder in children and adolescents. Pediatrics 2011;128:1007-1022.

10. Cohen SC, Mulqueen JM, Ferracioli-Oda E, Stuckelman ZD, Coughlin CG, Leckman JF, et al. Meta-analysis: risk of tics associated with psychostimulant use in randomized, placebo-controlled trials. J Am Acad Child Adolesc Psychiatry 2015;54:728-736.

11. Bloch MH, Panza KE, Landeros-Weisenberger A, Leckman JF. Metaanalysis: treatment of attention-deficit/hyperactivity disorder in children with comorbid tic disorders. J Am Acad Child Adolesc Psychiatry 2009;48:884-893.

12. Leckman JF. Tourette's syndrome. Lancet 2002;360:1577-1586.

13. Robertson MM. Tourette syndrome, associated conditions and the complexities of treatment. Brain 2000;123:425-462.

14. Mills S, Hedderly T. A guide to childhood motor stereotypies, tic disorders and the tourette spectrum for the primary care practitioner. Ulster Med J 2014;83:22-30.

15. So YK, Noh JS, Kim YS, Ko SG, Koh YJ. The reliability and validity of Korean parent and teacher ADHD rating scale. J Korean Neuropsychiatr Assoc 2002;41:283-289.

16. Simonoff E, Taylor E, Baird G, Bernard S, Chadwick O, Liang H, et al. Randomized controlled double-blind trial of optimal dose methylphenidate in children and adolescents with severe attention deficit hyperactivity disorder and intellectual disability. J Child Psychol Psychiatry 2013;54:527-535.

17. Aman MG, Buican B, Arnold LE. Methylphenidate treatment in children with borderline IQ and mental retardation: analysis of three aggregated studies. J Child Adolesc Psychopharmacol 2003;13:29-40.

18. Coutton C, Dieterich K, Satre V, Vieville G, Amblard F, David M, et al. Array-CGH in children with mild intellectual disability: a populationbased study. Eur J Pediatr 2015;174:75-83.

19. des Portes V, Livet MO, Vallée L. [A practical diagnostic approach to mental deficiency in 2002]. Arch Pediatr 2002;9:709-725.
20. Pringsheim T, Okun MS, Müller-Vahl K, Martino D, Jankovic J, Cavanna AE, et al. Practice guideline recommendations summary: Treatment of tics in people with Tourette syndrome and chronic tic disorders. Neurology 2019;92:896-906

21. Gaub M, Carlson CL. Gender differences in ADHD: a meta-analysis and critical review. J Am Acad Child Adolesc Psychiatry 1997;36:10361045.

22. Hallett M. Tourette Syndrome: Update. Brain Dev 2015;37:651-655.

23. Nøvik TS, Hervas A, Ralston SJ, Dalsgaard S, Rodrigues Pereira R, Lorenzo MJ. Influence of gender on attention-deficit/hyperactivity disorder in Europe--ADORE. Eur Child Adolesc Psychiatry 2006;15(Suppl1): I15-I24.

24. Ramtekkar UP, Reiersen AM, Todorov AA, Todd RD. Sex and age differences in attention-deficit/hyperactivity disorder symptoms and diagnoses: implications for DSM-V and ICD-11. J Am Acad Child Adolesc Psychiatry 2010;49:217-228.e211-213.

25. Willcutt EG. The prevalence of DSM-IV attention-deficit/hyperactivity disorder: a meta-analytic review. Neurotherapeutics 2012;9:490-499.

26. Andersen SL. Stimulants and the developing brain. Trends Pharmacol Sci 2005;26:237-243.

27. Gadow KD, Sverd J, Nolan EE, Sprafkin J, Schneider J. Immediate-release methylphenidate for ADHD in children with comorbid chronic multiple tic disorder. J Am Acad Child Adolesc Psychiatry 2007;46:840848.

28. Jankovic J, Kurlan R. Tourette syndrome: evolving concepts. Mov Disord 2011;26:1149-1156.

29. Banaschewski T, Becker K, Döpfner M, Holtmann M, Rösler M, Romanos M. Attention-Deficit/Hyperactivity Disorder. Dtsch Arztebl Int 2017;114:149-159.

30. Reale L, Bartoli B, Cartabia M, Zanetti M, Costantino MA, Canevini MP, et al. Comorbidity prevalence and treatment outcome in children and adolescents with ADHD. Eur Child Adolesc Psychiatry 2017;26:14431457.

31. Bloch MH, Leckman JF. Clinical course of Tourette syndrome. J Psychosom Res 2009;67:497-501. 


\section{Supplementary Materials}

\section{A. Extended psychiatric family histories in non-tic aggravation group}

In non-tic aggravation group, there were 17 subjects who had psychiatric family history, such as 3 parents' ADHD, 7 parents' depressive disorder, 1 parent's bipolar disorder, 1 parents' adjustment disorder, 1 grandparents' depressive disorder, 1 grandparents' bipolar disorder, 1 grandparents' dementia, 2 siblings' ADHD, 1 siblings' bipolar disorder and 1 siblings' autism.

\section{B. Extended comorbidities in tic aggravation group and non-tic aggravation group}

In tic aggravation group, there were 6 subjects who had comorbidities, such as 5 anxiety disorder, 1 disruptive mood dysregulation disorder, 1 language disorder, 1 communication disorder and 1 learning disorder.

In non-tic aggravation group, there were 40 subjects who had comorbidities, such as 11 anxiety disorder, 10 oppositional defiant disorder, 8 depressive disorder, 8 bipolar disorder, 4 obsessive-compulsive disorder, 4 communication disorder, 2 stressor related disorder, 1 conduct disorder and 1 enuresis. 


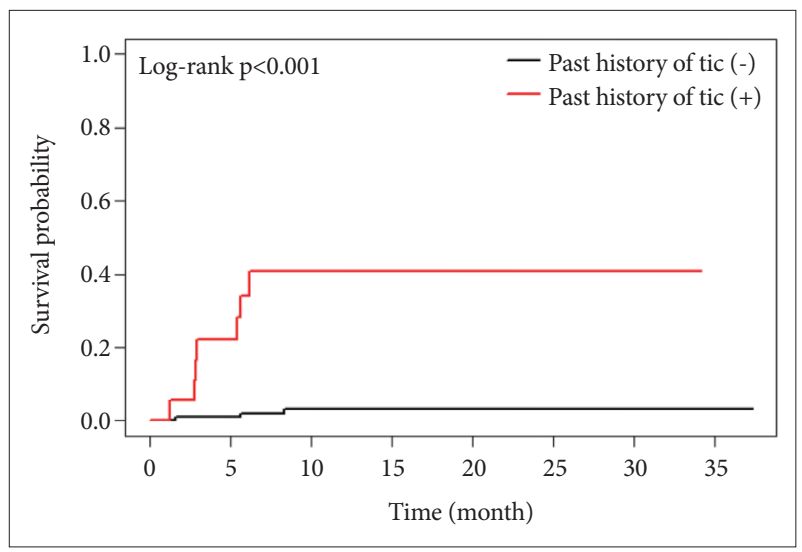

\begin{tabular}{|c|c|c|c|c|c|c|}
\hline \multicolumn{7}{|c|}{ Past history of tic (-) } \\
\hline Time & n.risk & n.event & Survival & Std. err & \multicolumn{2}{|c|}{$95 \%$ CI of survival rate } \\
\hline 1.61 & 103 & 1 & 0.99 & 0.00966 & 0.972 & 1 \\
\hline 5.61 & 92 & 1 & 0.98 & 0.01435 & 0.952 & 1 \\
\hline 8.33 & 76 & 1 & 0.967 & 0.01909 & 0.93 & 1 \\
\hline \multicolumn{7}{|c|}{ Past history of tic (+) } \\
\hline Time & n.risk & n.event & Survival & Std. err & \multicolumn{2}{|c|}{$95 \%$ CI of survival rate } \\
\hline 1.25 & 18 & 1 & 0.944 & 0.054 & 0.844 & 1 \\
\hline 2.79 & 17 & 1 & 0.889 & 0.0741 & 0.755 & 1 \\
\hline 2.85 & 16 & 1 & 0.833 & 0.0878 & 0.678 & 1 \\
\hline 2.95 & 15 & 1 & 0.778 & 0.098 & 0.608 & 0.996 \\
\hline 5.41 & 13 & 1 & 0.718 & 0.1072 & 0.536 & 0.962 \\
\hline 5.64 & 12 & 1 & 0.658 & 0.1137 & 0.469 & 0.923 \\
\hline 6.16 & 10 & 1 & 0.592 & 0.1199 & 0.398 & 0.881 \\
\hline
\end{tabular}

Supplementary Figure 1. Time to tic aggravation and number at risk with Kaplan-Meier curve. 